\title{
Bending Actuators with Maximum Curvature and Force and Zero Interfacial Stress
}

\author{
BENJAMIN SHAPIRO ${ }^{2, *}$ AND ELISABETH SMELA ${ }^{1}$ \\ ${ }^{1}$ Mechanical Engineering Department, University of Maryland \\ College Park, MD 20742, USA \\ ${ }^{2}$ Aerospace Engineering Department, University of Maryland \\ College Park, MD 20742, USA
}

\begin{abstract}
Stress at the interface of a bilayer actuator can lead to delamination, and this stress increases with the difference in the Young's moduli of the two layers. In this study, an actuator that includes a third 'buffer' layer is modeled, and it is shown how optimization of the thickness and modulus of this layer can reduce stress at that interface to zero, with no loss of curvature and only a small loss in force. A polypyrrole (PPy)/conducting polymer/gold trilayer is used as a model system, with the aim of reducing stress at the weak polymer/gold interface. For a $450 \mathrm{~nm}$ thick PPy layer with a Young's modulus of $0.2 \mathrm{GPa}$, an intermediate layer of $150 \mathrm{~nm}$ thickness with a modulus of $5 \mathrm{GPa}$ reduces the stress to zero with no loss of curvature and only a $7 \%$ loss in force compared to the bilayer case.
\end{abstract}

Key Words: actuator, polypyrrole, delamination, bilayer, stress.

\section{INTRODUCTION}

A CTUATORS made by laminating dissimilar materials suffer the risk of delamination. Conjugated polymer actuators, for example, require contact with a metal electrode throughout their length in order to undergo full actuation ( $\mathrm{Lu}$ et al., 2001; Smela et al., 2005), yet, since there are no covalent bonds between the polymer and the metal, this interface is weak. The large cyclic interfacial stresses caused by repeated expansion and contraction of the polymer sooner or later result in adhesive failure.

This problem has been mitigated primarily through mechanical interlocking (Pyo et al., 2003; Liu et al., 2005) or embedding (Ding et al., 2003) of the electrode. These solutions, however, are not always applicable. Therefore this article presents an approach to reduce the stress that involves the deposition of an intermediate conducting layer, such as a conjugated polymer that does not undergo significant volume change. The highest-stress interface becomes the strong one between the two polymers, which can be covalently bound. A trilayer model is presented, and it is shown how the thickness and Young's modulus of the buffer layer impact the stress at the gold interface, the actuator curvature and the force.

*Author to whom correspondence should be addressed. E-mail: benshap@eng.umd.edu

\section{MODEL FOR CURVATURE, MOMENT, AND STRESS IN BEAM ACTUATORS}

The model is based on the classical beam theory (Gere and Timoshenko, 1990) which assumes that: the thickness of the beam is small compared to the minimum radius of curvature undergone during actuation $(\mathrm{h}<<\mathrm{R})$; there is a linear material relationship between stress and strain; and the Young's modulus of elasticity, $E$, and the actuation coefficient of expansion in the polymer, $\alpha$, are constant and do not depend on spatial location inside each layer. For the bilayer case, this yields Timoshenko's classical model for metal bimorphs that bend upon heating due to different coefficients of thermal expansion (Timoshenko, 1925). Timoshenko's thermal expansion coefficient per unit temperature has been replaced by a total polymer expansion coefficient, and the model has been extended to three layers: actuating polymer, buffer polymer, and gold.

The Timoshenko model in the context of a bilayer is briefly summarized. As a bilayer beam bends, the two initially parallel planes indicated by $A B$ and $\mathrm{CD}$ in Figure 1 are tilted relative to one another. At some vertical location, denoted by PQ, there is no strain. All locations above PQ experience stretching (positive strain) proportional to the vertical distance from $\mathrm{PQ}$, and all locations below PQ experience compression (negative strain). Thus, the equation for the strain $\varepsilon$ due to bending is linear in the height variable $z: \varepsilon(z)=\varepsilon_{0}+z / R=\varepsilon_{0}+\kappa z$, where $\varepsilon_{0}$ is the strain 
(a) Line of zero

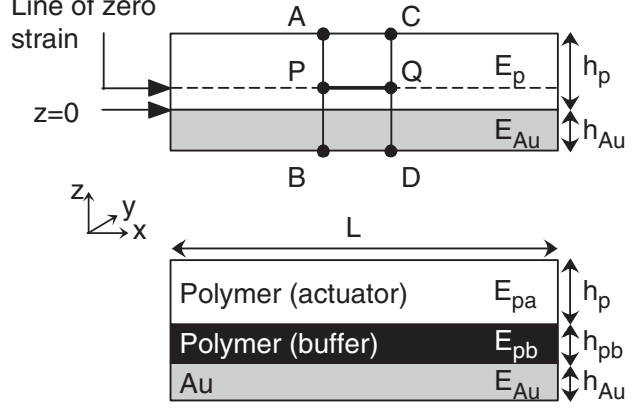

(b)

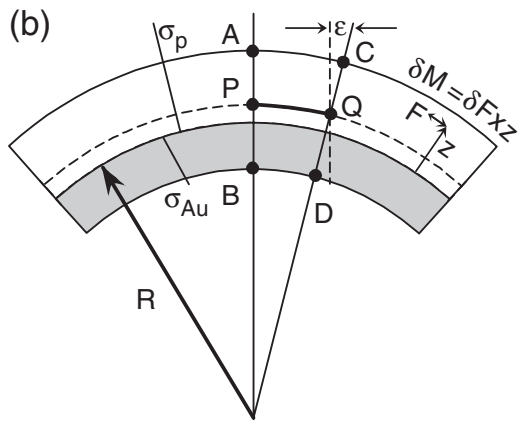

Figure 1. (a) A straight and (b) bent polymer/gold bilayer beam showing definitions of the variables. Strain $\varepsilon(z)$ is continuous across the interface, but the stress $\sigma(z)=E_{j}\left(\varepsilon(z)-\alpha_{j}\right)$ is not. The moment $\delta M$ produced by each unit volume of polymer is the force $\delta F$ it generates times its moment arm $z$ here defined from the polymer/gold interface. (c) A trilayer beam with a polymer buffer layer.

at the interface $z=0, R$ is the radius of curvature of the beam, and $\kappa=1 / R$ is the curvature. This result is purely geometric and holds for beams of any combination of materials and for bending due to either external forces or actuation-induced curvature. The modeling task is to determine the radius of curvature $R$ and the strain at the polymer/gold interface $\varepsilon_{0}$ : these two numbers depend on the beam geometry and material properties. This will then completely describe the deformation, moment, strain, and stress in the beam.

As described previously (Timoshenko, 1925, Christophersen et al., 2005), the parameters $\varepsilon_{0}$ and $\kappa$ are found by setting the net force and moment to zero when the polymer undergoes actuation. The strain $\alpha$ is the free actuation strain of the active polymer layer when it is not attached to the gold. Solving the resulting set of equations gives

$$
\varepsilon_{0}=\left(\frac{m n\left(4+3 m+m^{3} n\right)}{1+4 m n+6 m^{2} n+4 m^{3} n+m^{4} n^{2}}\right) \alpha
$$

where $m$ is the ratio of film thicknesses, $m=h_{\mathrm{p}} / h_{\mathrm{Au}}$, and $n$ is the ratio of their Young's moduli, $n=E_{\mathrm{p}} / E_{\mathrm{Au}}$, and

$$
\kappa=\frac{1}{R}=\frac{\alpha}{h_{\mathrm{Au}}}\left(\frac{6 m n(1+m)}{1+4 m n+6 m^{2} n+4 m^{3} n+m^{4} n^{2}}\right) \alpha .
$$

This is easily extended to a trilayer beam
As discussed previously, delamination at the polymer/gold interface is caused by stresses at that interface. The stress at the polymer/gold interface $\sigma_{i}$ for the bilayer is the difference between the stress in the polymer just above the interface, at $z=0^{+}$, and the stress in the gold just below the interface, at $z=0^{-}$. The stress in the polymer is given by $\sigma=E_{\mathrm{pa}}(\varepsilon-\alpha)$. The stress in the gold is simply $\sigma=E_{\mathrm{Au}} \varepsilon$, since there is no actuation strain. This gives

$$
\begin{aligned}
\sigma_{i} & =\sigma\left(z=0^{-}\right)-\sigma\left(z=0^{+}\right) \\
& =E_{\mathrm{Au}}\left(\varepsilon\left(0^{-}\right)-\alpha\right)-E_{\mathrm{pa}} \varepsilon\left(0^{+}\right) \\
& =\left(E_{\mathrm{Au}}-E_{\mathrm{pa}}\right) \varepsilon_{0}-E_{\mathrm{pa}} \alpha .
\end{aligned}
$$

The interfacial stress depends primarily on the difference in the Young's moduli of the layers, and, as shown in Figure 1, it is not continuous across the interface. Note that in the trilayer case, the interfacial stress at the gold surface is given simply by

$$
\sigma_{i}=\left(E_{\mathrm{Au}}-E_{\mathrm{pb}}\right) \varepsilon_{0}
$$

because the buffer layer does not undergo actuation.

When actuated, a bi- or tri-layer beam will produce a net torque or moment. The blocked moment $M$ per unit beam width, i.e., the amount of torque that is

$$
\begin{gathered}
\varepsilon_{0}=\frac{\left(m_{\mathrm{a}} n_{\mathrm{a}}\left(4+6 m_{\mathrm{b}}+3 m_{\mathrm{a}}-2 m_{\mathrm{b}}^{3} n_{\mathrm{b}}-3 m_{\mathrm{b}}^{2} m_{\mathrm{a}} n_{\mathrm{b}}+m_{\mathrm{a}}^{3} n_{\mathrm{a}}\right) \alpha\right.}{\left(\begin{array}{l}
1+m_{\mathrm{b}}^{4} n_{\mathrm{b}}^{2}+4 m_{\mathrm{a}} n_{\mathrm{a}}+6 m_{\mathrm{a}}^{2} n_{\mathrm{a}}+4 m_{\mathrm{a}}^{3} n_{\mathrm{a}}+m_{\mathrm{a}}^{4} n_{\mathrm{a}}^{2}+4 m_{\mathrm{b}}^{3}\left(n_{\mathrm{b}}+m_{\mathrm{a}} n_{\mathrm{b}} n_{\mathrm{a}}\right) \\
+6 m_{\mathrm{b}}^{2}\left(n_{\mathrm{b}}+2 m_{\mathrm{a}} n_{\mathrm{a}}+m_{\mathrm{a}}^{2} n_{\mathrm{b}} n_{\mathrm{a}}\right)+4 m_{\mathrm{b}}\left(n_{\mathrm{b}}+3 m_{\mathrm{a}}\left(1+m_{\mathrm{a}}\right) n_{\mathrm{a}}+m_{\mathrm{a}}^{3} n_{\mathrm{b}} n_{\mathrm{a}}\right.
\end{array}\right)} \\
\kappa=\frac{1}{R}=\frac{6 m_{\mathrm{a}} n_{\mathrm{a}}\left(1+m_{\mathrm{a}}+m_{\mathrm{b}}^{2} n_{\mathrm{b}}+m_{\mathrm{b}}\left(2+m_{\mathrm{a}} n_{\mathrm{b}}\right)\right) \alpha}{h_{\mathrm{Au}}\left(\begin{array}{l}
1+m_{\mathrm{b}}^{4} n_{\mathrm{b}}^{2}+4 m_{\mathrm{a}} n_{\mathrm{a}}+6 m_{\mathrm{a}}^{2} n_{\mathrm{a}}+4 m_{\mathrm{a}}^{3} n_{\mathrm{a}}+m_{\mathrm{a}}^{4} n_{\mathrm{a}}^{2}+4 m_{\mathrm{b}}^{3}\left(n_{\mathrm{b}}+m_{\mathrm{a}} n_{\mathrm{b}} n_{\mathrm{a}}\right) \\
+6 m_{\mathrm{b}}^{2}\left(n_{\mathrm{b}}+2 m_{\mathrm{a}} n_{\mathrm{a}}+m_{\mathrm{a}}^{2} n_{\mathrm{b}} n_{\mathrm{a}}\right)+4 m_{\mathrm{b}}\left(n_{\mathrm{b}}+3 m_{\mathrm{a}}\left(1+m_{\mathrm{a}}\right) n_{\mathrm{a}}+m_{\mathrm{a}}^{3} n_{\mathrm{b}} n_{\mathrm{a}}\right)
\end{array}\right)}
\end{gathered}
$$

where $m_{a}=h_{\mathrm{pa}} / h_{\mathrm{Au}}, \quad n_{\mathrm{a}}=E_{\mathrm{pa}} / E_{\mathrm{Au}}$ and $m_{\mathrm{b}}=h_{\mathrm{pb}} / h_{\mathrm{Au}}$, $n_{\mathrm{b}}=E_{\mathrm{pb}} / E_{\mathrm{Au}}$. The trilayer Equations (3) and (4) reduce to Equations (1) and (2) when $m_{\mathrm{b}}$ is set to zero. (The Mathematica script that generated the results in this article is available upon request from the authors.) required to keep the beam straight when the beam is actuated, is given by

$$
M=\frac{E_{\mathrm{Au}} h_{\mathrm{Au}}^{2} m n(1+m) \alpha}{2(1+m n)}
$$


for the bilayer beam and

$$
M=\frac{E_{\mathrm{Au}} h_{\mathrm{Au}}^{2} m_{\mathrm{a}}\left(1+m_{\mathrm{a}}+m_{\mathrm{b}}^{2} n_{\mathrm{b}}+m_{\mathrm{b}} n_{\mathrm{a}}\left(2+m_{\mathrm{a}} n_{\mathrm{b}}\right)\right) \alpha}{2\left(1+m_{\mathrm{b}} n_{\mathrm{b}}+m_{\mathrm{a}} n_{\mathrm{a}}\right)}
$$

for the trilayer beam. (Note that since $M$ is the moment per unit width, it has units of Newtons.)

The moment is constant throughout the length of the beam. If the deflection at the tip of the beam is blocked by a force applied at the tip, then there is a relation between required blocked force and the blocked moment: $F=3 w M / 2 L$ (Gere, 1990), where $w$ is the width of the beam. (The reason for the factor of $3 / 2$ is that the blocked force at the tip creates a varying moment throughout the length of the beam since the moment arm varies from 0 at the tip to $L$ at the root.) In this case the beam will buckle slightly, but its tip will remain stationary.

\section{THE BILAYER BEAM}

First, a review is provided of the curvature (which determines deflection) and moment (which determines strength) of a bilayer beam as a function of polymer thickness. Results are shown for parameters that match literature values and are appropriate for thin, microfabricated actuators (Christophersen et al., 2005): the gold thickness is $h_{\mathrm{Au}}=100 \mathrm{~nm}$ and the polymer thickness is varied as $h_{\mathrm{p}}=m h_{\mathrm{Au}}$; the Young's modulus of the gold and polymer are approximately $E_{\mathrm{Au}}=83 \mathrm{GPa}$ and $E_{\mathrm{p}}=0.2 \mathrm{GPa} ; n=E_{\mathrm{p}} / E_{\mathrm{Au}}$; and the free expansion of the polymer (when it is not attached to the gold) is on the order of $\alpha=5 \%$. Figure 2 shows the curvature of the beam $\kappa$ and the blocked moment $M$ as a function of polymer to gold thickness ratio.

When the polymer thickness is zero, both $\kappa$ and $M$ are zero. The curvature initially increases as the polymer thickness is increased, but eventually, once the polymer thickness exceeds approximately five times the thickness of the gold with these parameters, the curvature decreases in $m$. This is because a very thick layer of polymer, even if it is active, will prevent the beam from bending effectively. The optimal $m$ that achieves the highest curvature is $m_{\mathrm{opt}}=5.5$. The behavior of the blocked moment is simpler: the strength increases monotonically with PPy thickness. The curve is nonlinear because as $m$ increases, not only does the amount of polymer increase, but it acts through an increasing vertical moment arm $h_{\mathrm{p}}$. Comparisons with experimental data show that this model is appropriate, but that the actuation strain $\alpha$ is $z$-dependent (Christophersen et al., 2005). For simplicity, $\alpha$ is treated as a constant in this study.

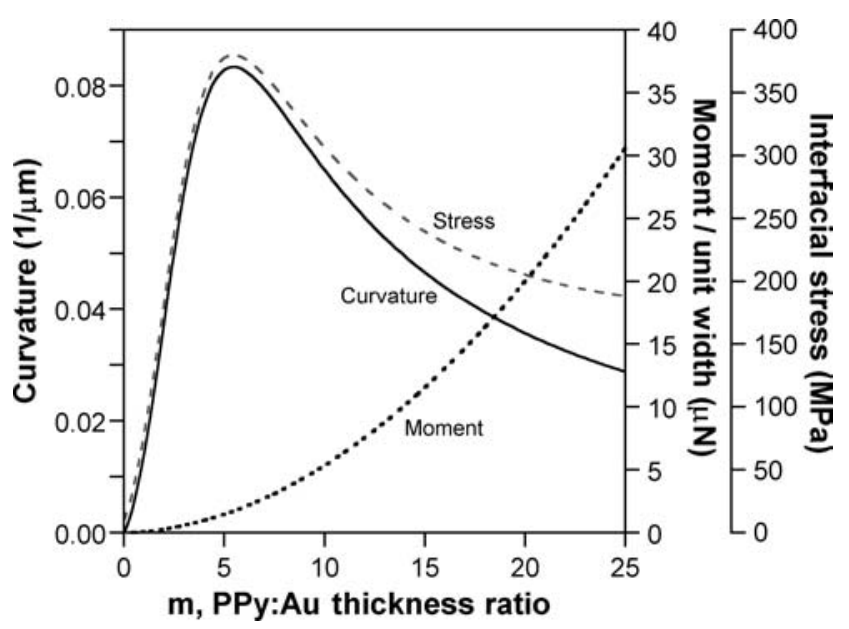

Figure 2. Beam curvature $\kappa$, blocked moment $M$ per unit beam width, and interfacial stress for a bilayer beam as a function of the polymer to gold thickness ratio $m=h_{p} / h_{A u}$. The remaining beam parameters are: $h_{A u}=100 \mathrm{~nm}, E_{p}=0.2 \mathrm{GPa}, E_{A u}=83 \mathrm{GPa}$, and $\alpha=5 \%$.

The interfacial stress closely follows the beam curvature: when the curvature is large, there is a large deformation and hence a high stress at the interface. Interfacial stresses are on the order of hundreds of MPa, which explains the severity of the delamination problem to be addressed.

\section{THE TRILAYER BEAM}

The addition of a middle, non-actuating, but electrically conductive layer of polymer is considered and the tradeoffs between curvature, moment, and interfacial stresses is examined. The rationale is that including a middle polymer layer will relieve much of the stress that occurs at the polymer/gold interface and will therefore minimize delamination. Stress between the polymer layers is not a concern because they are held together by covalent bonds, so this interface is able to withstand high stresses, unlike the polymer/gold interface, which has only weak van der Waals bonds.

To start, the curvature and stress in the trilayer beam, as a function of buffer layer thickness when the buffer and actuating polymers have the same Young's modulus are examined. Figure 3(a) shows $\kappa$ according to Equation (4) in terms of the active, actuating polymer thickness $h_{\mathrm{pa}}=m_{\mathrm{a}} h_{\mathrm{Au}}$ and the buffer polymer thickness $h_{\mathrm{pb}}=m_{\mathrm{b}} h_{\mathrm{Au}}$. The remaining beam parameters are held constant at their prior values. The surfaces of Figure 3 are colored in gray scale from maximum (black) to minimum (white) interfacial stress.

When $m_{\mathrm{b}}=0$ and there is no buffer layer, the curvature behavior is the same as that shown in Figure 2. As the buffer thickness $h_{\mathrm{pb}}$ increases, the curvature monotonically decreases. Note that the interfacial stress 
(a)

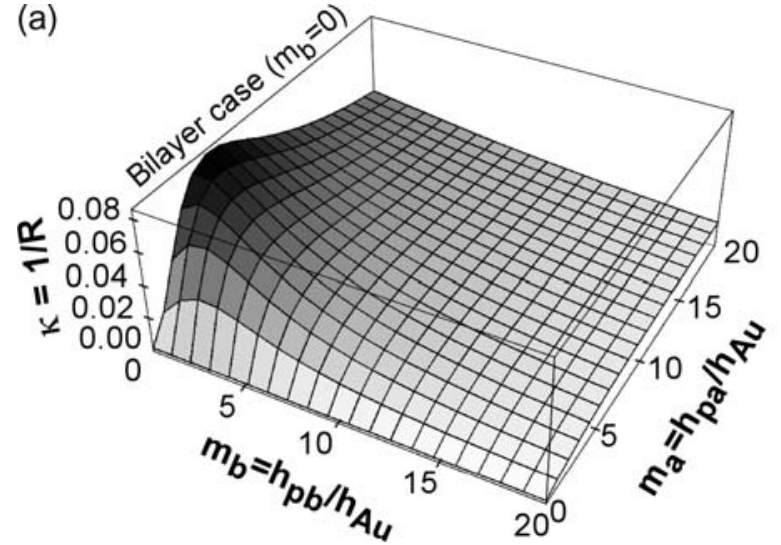

(b)

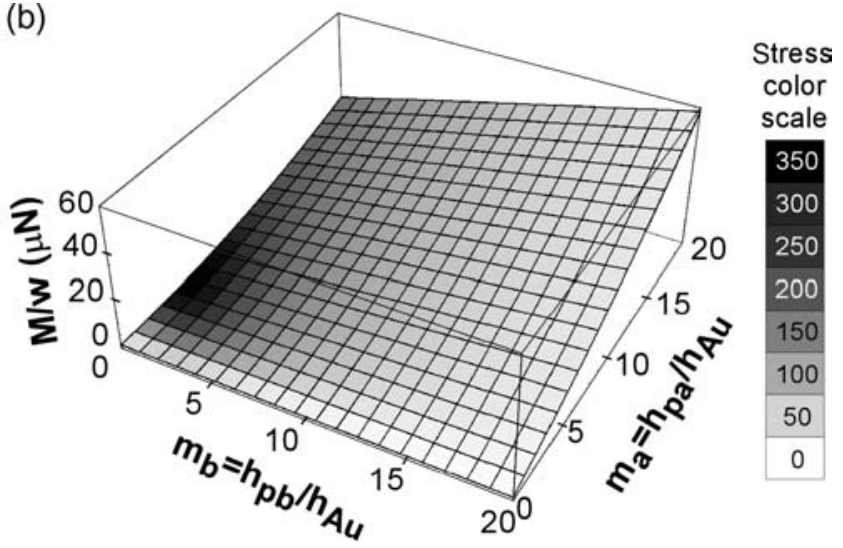

Figure 3. (a) Curvature $\kappa$ and (b) blocked moment $M$ per unit beam width for a trilayer beam in terms of thickness ratios $m_{a}$ and $m_{b}$. The surfaces are colored in gray scale by the interfacial stress $\sigma_{i}$. Black corresponds to a maximum stress of $350 \mathrm{MPa}$ and white to zero. $\left(h_{A u}=0.1 \mu \mathrm{m}\right.$, $E_{p a}=E_{p b}=0.2 \mathrm{GPa}$, and $\alpha=5 \%$.)

$\sigma_{\mathrm{i}}$ is highest at $m_{\mathrm{a}}=5.5, m_{\mathrm{b}}=0$, the maximum curvature design point for the bilayer beam, and it decreases sharply as $h_{\mathrm{pb}}$ increases. Holding all other beam parameters constant, it is possible to decrease the stress significantly, although with a loss of curvature, by including a buffer layer with the same stiffness as the active polymer layer.

Figure 3(b) shows the behavior of the blocked moment per unit beam width, according to Equation (8), as a function of the two thickness ratios. The strength of the beam increases as the thicknesses of both the actuating and buffer layers are increased. (Increases in both thicknesses create larger vertical moment arms.)

The large difference between the Young's modulus of gold $\left(E_{\mathrm{Au}}=83 \mathrm{GPa}\right)$ and that of the polymer $\left(E_{\mathrm{pa}}=0.2 \mathrm{GPa}\right)$, compounded by the high strain there, is what creates the high stresses at the interface. It is possible, by electrochemical deposition, to increase the stiffness of the middle polymer layer through the choice of dopant and polymerization conditions. Alternatively, a different conjugated polymer can be used. Values as high as $4 \mathrm{GPa}$ have been reported for PPy (Wallace et al., 1997). (The maximum reported modulus for a conjugated polymer was $100 \mathrm{GPa}$ in undoped, stretched, trans polyacetylene, but this material is not stable in air.) The buffer layer should be chosen not only so that it is stiffer than the actuating layer, but also so that it does not undergo any volume change upon electrochemical oxidation/reduction of the actuating layer, thereby minimizing the strain at the interface. By doing so, it should be possible to lower the stress at the polymer/gold interface significantly.

The previous discussion lays out the landscape of curvature, blocked moment, and interfacial stress behavior with respect to the beam parameters. Essentially, including a buffer polymer layer reduces the beam curvature (bad), reduces the interfacial stress (good), and increases the moment (good). One should be able to optimize the overall trilayer design by decreasing the thickness of the entire beam to recover the original bilayer curvature, while still keeping the low interfacial stress, 'paying' with the moment. The question, 'how to best achieve this?' is now answered.

Initially the $m_{\mathrm{a}}=h_{\mathrm{pa}} / h_{\mathrm{Au}}$ that achieves the maximal trilayer curvature, as a function of all the other beam parameters, is found. This involves differentiating $\kappa$ in Equation (4) with respect to $m_{\mathrm{a}}$, setting the derivative to zero, and solving for $m_{\mathrm{a}}$. Since Equation (4) has $m_{\mathrm{a}}$ squared in its numerator and $m_{\mathrm{a}}$ to the power fourth in its denominator, upon differentiation this gives a fifthorder equation for $m_{\mathrm{a}}\left(4+2=6\right.$ powers in $m_{\mathrm{a}}$ minus one power by differentiation), which has five roots. Only one solution is realistic (the others are negative or complex) and this optimal solution is plotted in Figure 4(a) as a function of $m_{\mathrm{b}}=h_{\mathrm{pb}} / h_{\mathrm{Au}}$ and $n_{\mathrm{p}}=E_{\mathrm{pb}} / E_{\mathrm{Au}}$ with all other beam parameters held constant. The analytical expression for $m_{\mathrm{a}}^{\mathrm{opt}}$ is lengthy (the algebraic result is a page long), so the formula is not presented here. For small values of $m_{\mathrm{b}}$ and $n_{\mathrm{b}}$, the optimal choice of $m_{\mathrm{a}}$ is between 5 and 6, as in Figure 2, but as these values increase, the optimal value drifts to higher numbers.

When the relative thickness $\left(m_{\mathrm{b}}\right)$ and stiffness $\left(n_{\mathrm{b}}\right)$ of the buffer layer is increased with constant gold thickness $h_{\mathrm{Au}}$, the curvature $\kappa$ of the beam decreases. The $h_{\mathrm{Au}}$ required to maintain the trilayer curvature equal to the highest bilayer curvature $\kappa_{b i}=83 \mathrm{~nm}^{-1}$ is now solved. This is done using the previous beam parameters and the optimal $m_{\mathrm{a}}^{\text {opt }}$. Figure 4(b) shows how the Au thickness must be reduced to maintain the desired curvature.

Now one can answer the design question: how to keep curvature and blocked moment at the bilayer maximum while significantly decreasing the polymer/gold interfacial stress? Figure 4(c) shows the dependence of the trilayer blocked moment and Figure 4(d) shows the interfacial stress. This set of figures should be understood as follows. For any choice of $m_{\mathrm{b}}$ and $n_{\mathrm{b}}$, choose the gold 

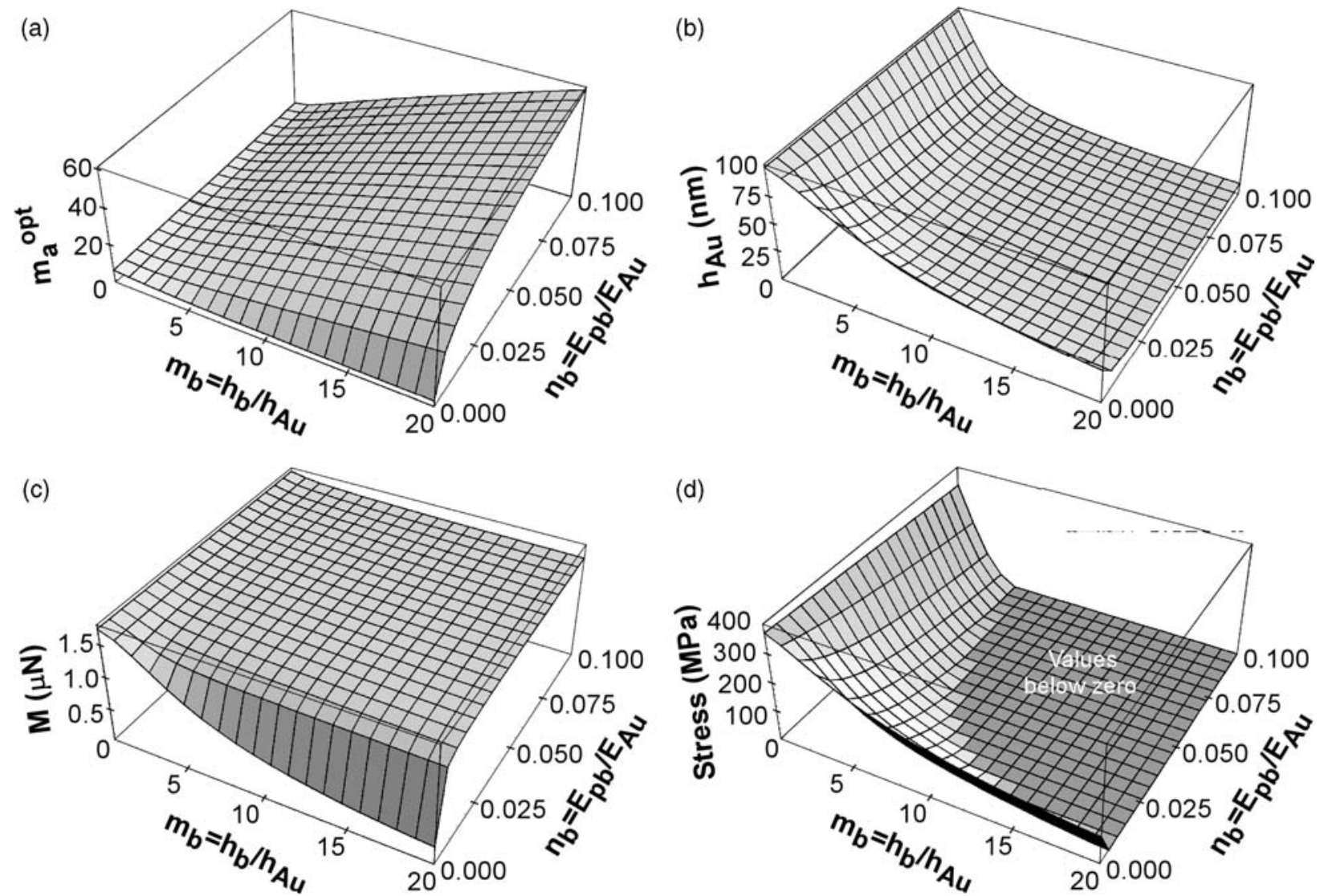

Figure 4. (a) The $m_{\mathrm{a}}^{\mathrm{opt}}=h_{p a} / h_{A u}$ ratio that maximizes trilayer beam curvature as a function of the thickness and stiffness ratios $m_{b}=h_{p b} / h_{A u}$ and $n_{b}=E_{p b} / E_{A u}$; (b) using $m_{\mathrm{a}}^{\mathrm{opt}}$, the corresponding gold thickness that should be used to keep the curvature of the trilayer beam equal to the maximum curvature of the bilayer beam; (c) the blocked moment for the trilayer beam with optimal $m_{\mathrm{a}}^{\mathrm{opt}}$ and $h_{A u}$; and (d) the corresponding interfacial stress, which goes through zero and changes sign. Other parameters held constant $\left(E_{A u}=83 \mathrm{GPa}, E_{p a}=0.2 \mathrm{GPa}\right.$, and $\left.\alpha=5 \%\right)$.
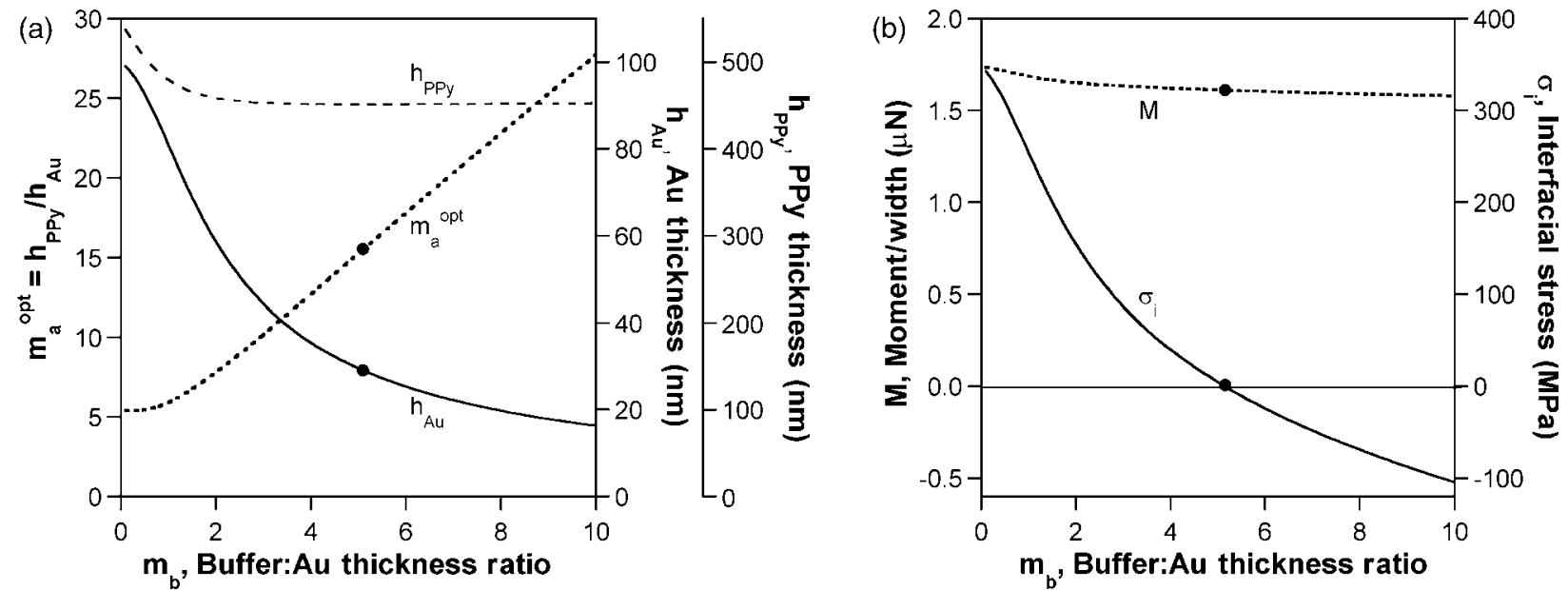

Figure 5. $2 D$ slices of variables in Figure 4 for $n_{b}=0.06$ : (a) $m_{\mathrm{a}}^{\text {opt }}$ and $h_{A u}$ and (b) $M$ and $\sigma_{i}$. Points mark the values to use to achieve zero interfacial stress.

thickness $h_{\mathrm{Au}}$ according to Figure 4(b). Next choose the actuator polymer thickness to be $h_{\mathrm{pa}}=m_{\mathrm{a}} h_{\mathrm{Au}}$ with $m_{\mathrm{a}}^{\text {opt }}$ taken from Figure 4(a). The resulting curvature of the beam will be $\kappa_{b i}=83 \mathrm{~nm}^{-1}$. The moment and interfacial stress will be as shown in Figure 4(c) and (d). (When $m_{\mathrm{b}}=0$, the bilayer case is recovered, where $m_{\mathrm{a}}^{\mathrm{opt}}=5.5$ and $M=1.74 \mu \mathrm{N}$.)
To obtain some specific numbers, this method is applied to the case $E_{\mathrm{pb}}=5 \mathrm{GPa}$ (so $n_{b}=0.06$ ), which, as discussed previously could be achieved with PPy, and it is assumed that the layer is deposited in a manner that leaves it conducting but without actuation strain. Figure 5 shows these 2D slices through the Figure 4 data. The optimum PPy thickness increases relative to 


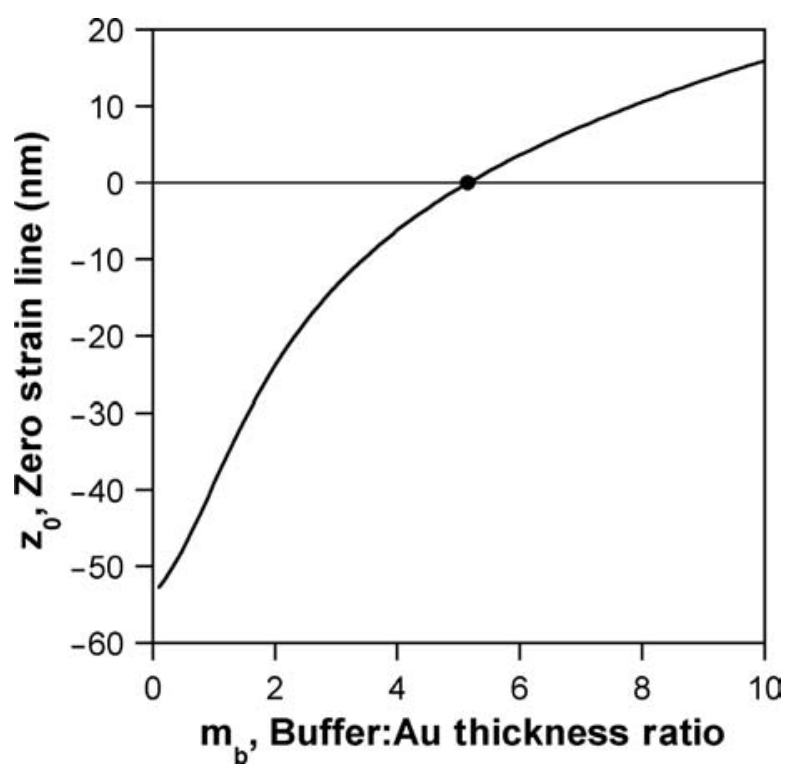

Figure 6. Position of the line of zero strain relative to the polymer/ gold interface.

the gold thickness because the latter must be reduced to maintain the bilayer curvature. The PPy thickness, however, does not change significantly. The moment decreases very little: it asymptotes to $1.5 \mu \mathrm{N}$, or $86 \%$ of the bilayer value. The stress drops rapidly with buffer layer thickness, hitting zero at a value of $m_{\mathrm{b}}=5.15$. At this point, the gold thickness is $30 \mathrm{~nm}$, the PPy thickness is $450 \mathrm{~nm}$, and the moment is $1.6 \mu \mathrm{N}$, or $93 \%$ of the bilayer value.

The reason for the drop in stress at the gold interface is that the insertion of a buffer layer moves the line of zero strain (Figure 1), as shown in Figure 6. It starts in the gold layer and moves upward into the polymer layer as $m_{\mathrm{b}}$ increases. At $m_{\mathrm{b}}=5.15$, it is right at the polymer/ gold interface.

\section{CONCLUSIONS}

By including a middle buffer layer it is possible to create a trilayer actuating polymer/buffer polymer/gold beam that will achieve the same curvature and almost the same moment as a bilayer beam, but with zero interfacial stress. This will eliminate the most significant cause of delamination. This article presented a design method to achieve such a trilayer beam design.

\section{REFERENCES}

Christophersen, M., Liu, Y., Shapiro, B. and Smela, E. 2005. "Characterization and Modeling of PPy Bilayer Microactuators. Part 1: Curvature," Sens. Act. B. (in press).

Ding, J., Liu, L., Spinks, G.M., Zhou, D.Z., Wallace, G.G. and Gillespie, J. 2003. "High Performance Conducting Polymer Actuators Utilising a Tubular Geometry and Helical Wire Interconnects," Synth. Met., 138(3):391-398.

Gere, J.M. and Timoshenko, S.P. 1990. Mechanics of Materials, PWSKENT Publishing Company, Boston.

Liu, Y., Gan, Q., Baig, S. and Smela, E. 2005. "Improving Adhesion of Polypyrrole to Gold for Long-term Actuation," In: SPIE 12th Annual Int'l. Symposium on Smart Structures and Materials, EAPAD, San Diego, CA, March 6-10.

Lu, W., Smela, E. and Mattes, B.R. 2001. "Electrochemical Actuation of Gilded Polyaniline Bilayers in Aqueous Acid Solutions," In: Proc. SPIE's 8th Int. Symp. Smart Struc. Mater., Electroactive Polymer Actuators and Devices (EAPAD), Newport Beach, CA, March 5-8, 4329:505-515.

Pyo, M., Bohn, C.C., Smela, E., Reynolds, J.R. and Brennan, A.B. 2003. "Direct Strain Measurement of Polypyrrole Actuators Controlled by the Polymer/Gold Interface," Chem. Mater., 15(4):916-922.

Smela, E., Lu, W. and Mattes, B.R. 2005. "Polyaniline Actuators, Part 1: PANI(AMPS) in HCl," Synth. Met., 151(1):25-42.

Timoshenko, S. 1925. "Analysis of Bi-metal Thermostats," J. Opt. Soc. Am., 11:233-256.

Wallace, G.G., Spinks, G.M. and Teasdale, P.R. 1997. Conductive Electroactive Polymers: Intelligent Materials Systems, Technomic Publishing Co., Lancaster. 